\title{
FACE AND FINGERPRINT FUSION SYSTEM FOR IDENTITY AUTHENTICATION USING FUSION Classifiers
}

\author{
Somashekhar ${ }^{1}$ B M and Y.S.Nijagunarya ${ }^{2}$ \\ ${ }^{1}$ Department of Information Science and Engineering ,Maharaja Institute of Technology \\ Mysore \\ ${ }^{2}$ Dept of Computer Science and Engineering, Siddaganga Institute of Technology \\ Tumkur
}

\begin{abstract}
In this work, we propose a feature level fusion and decision level fusion of face and fingerprint for designing a multimodal biometric system. Initially, Gabor and Scale Invariant Feature Transform features are extracted for both offline face and fingerprint of a person and studied the identification accuracy. Later the fusion of the biometric traits is recommended at feature level using all possible combinations of feature vectors. The possible combination of features is fed into fusion classifier of K-Nearest Neighbour(KNN), Support Vector Machine (SVM), Navie Bayes(NB) and Radial Basis Function(RBF). The best combination of feature vectors and fusion classifiers is identified for the proposed multimodal biometric system. Experiments are conducted on Face database and fingerprint database to assess the actualadvantage of the fusion of these biometric traits, in comparison to the unimodal biometric system. Experimental results reveal that fusion combination outperforms individual.
\end{abstract}

\section{INTRODUCTION}

Biometric systems using a single biometric trait either for identification or for verification is called unimodal biometric system. A unimodal biometric system sometimes fails to be accurate enough for the identification of a large user population due to problems such as noisy data, intraclass variations, restricted degrees of freedom, non-universality, spoof attacks, and unacceptable error rates $(\mathrm{Ko},[1])$. So many researchers have recommended usage of multiple biometric traits as a remedy (Ko, [1]; Ross and Jain [2]). One of the basic requirements of any biometric trait is its universality. However, no biometric trait is truly universal. In other words, no single biometric trait is ideal but a number of them are admissible (Ross et al., [3]). So the problems associated with unimodal biometric systems can be overcome by multibiometric systems. A multibiometric system unifies the information presented by multiple biometric sources. Multiple biometric sources include multiple sensors, multiple instances, multiple samples, multiple algorithms, and/or multiple biometric traits. Though the problem of noisy data can be overcome by the use of multiple sensors for the same biometric trait, the other potential problems associated with unimodal biometric systems will remain. The problem of spoofing can be reduced by multiple instances. It ensures the presence of a user by asking the user to provide a random subset of biometric measurements. Even multi-sample or multi-algorithmic biometric systems improve the overall performance of the biometric system, the problems of unimodal biometric systems still remain. As a remedy, multimodal biometric systems that use different biometric traits can be expected to be more effective. 
International Journal of Computer Science \& Engineering Survey (IJCSES) Vol.9, No.1/2/3, August 2018

Multimodal biometric systems have several advantages over unimodal biometric systems.A multimodal system offers increased performance, anti-spoofing, acceptable error rates, and the ability to operate on a large user population. Multimodal biometric systems acquire more than one type of information and so it offers a substantial improvement in the matching accuracy as compared to that of unimodal system. Multimodal biometric systems are capable of addressing the non universality issue with respect to unimodal biometric system. If any user fails to possess a single valid biometric trait still they can be enrolled into a system using another valid biometric trait. In some situations, somebiometric systems cannot properly work for every user. For example, a mute personcannot use a voice recognition system or a person with missing fingers cannot use thefingerprint identification system. Moreover, multimodal biometric systems offer anincrease in anti-spoofing by making it more difficult for an imposter to fool the systembecause imposter has to forge multiple biometric traits and probably within a certain timelimit. These biometric systems are insensitive to the noise on the sensed data. That is, when information acquired from a single biometric trait is corrupted by noise we can use another trait of the same user to perform the task. Multimodal biometric systems can also provide the capability to search a large database in an efficient and fast manner.

This can be achieved by a relatively simple but less accurate modality to prune the database before using the more complex and accurate modality on the remaining data to perform the final identification task. However, an effective fusion strategy is necessary to combine the information presented by multiple sources of biometric information. Based on the type of information available, fusion can occur at the sensor level, feature level, match score level, and decision level. Sensor level fusion entails the consolidation of evidence presented by multiple sources of raw data before they are subjected to feature extraction. Feature level fusion involves consolidating the information presented by two or more biometric feature sets of the same user. The scores generated by multiple classifiers pertaining to different modalities are combined in match-score level fusion. The final decisions of multiple classifiers are consolidated in decision level fusion. Along these levels the biometric information is gradually extracted and reduced. On the first two stages, the information content is rich, but in most cases noisy and redundant. On the other hand, matching score level, theinformation is reduced into a single quantity, indicating the likelihood that the biometric data belongs to a certain class. In decision level, the information is further reduced to the discrete class labels. In literature, very few research issues are reported on sensor level fusion (Heo et al., [4]; Singh et al., [5]; Singh et al., [6]). The main interest in sensor level fusion lies in multi-sample system that captures multiple snapshots of the same biometric. Thus, in literature most of the works reported on sensor level fusion are with the application of fusing visible and thermal face image. Heo et al., [4] proposed a weighted image fusion of visible and thermal face images where weights are assigned empirically on the visible and thermal face images by decomposing them using wavelet transform. Singh et al., [6] employed a genetic algorithm for feature selection and fusion where group of wavelet features from visible and thermal face images are selected and fused to form a single image and here there is no scope for weighting. Singh et al., [5] proposed a weighted image fusion using $2 \mathrm{v}$ - Granular support vector machine where weights are assigned by finding the activity level of visible and thermal face image. Recently, Kisku et al., [7] proposed a sensor level fusion scheme for face and palmprint. Finally, inverse wavelet transform is carried out on this fused image of face and palmprint. Then, feature extraction is carried out on this fused image using scale invariant feature transform (SIFT) technique to make the decision. Feature level fusion involves consolidating the evidences presented by two or more biometric feature sets of the same individual.

Thus as compared to match score level or decision level fusion, the feature level fusion exhibits a rich set of information. In performing the feature level fusion either we can use same feature extraction algorithm (Yao et al., [8]; Jing et al., [9]; Xiuqin et al., 2008; Rattani and Tistarelli, [11]) or different feature extraction algorithms (Feng et al., [12]; Zhou and Bhanu, [13]; Ross and 
International Journal of Computer Science \& Engineering Survey (IJCSES) Vol.9, No.1/2/3, August 2018

Govindrajan, [14]) on different modalities whose features have to be fused. The feature level fusion is challenging because, relationship between features is not known, and structurally incompatible features are common in addition to the curse of dimensionality. Because of these difficulties, only limited work is reported on feature level fusion of multimodal biometric system. The majority of the work reported on feature level fusion is related to multimodal biometric systems using face and palmprint.

Feng et al., [15] proposed the feature level fusion of face and palmprint in which principle component analysis (PCA) and independent component analysis (ICA) are used for feature extraction. Further, feature concatenation is carried out to obtain the fused features of face and palmprint. Yao et al., [8] also proposed a multimodal biometric system using face and palmprint at feature level. Here, Gabor features of face and palmprints are obtained individually. Extracted Gabor features are then analyzed using linear projection scheme such as PCA to obtain the dominant principal components of faces and palmprints separately. Finally, feature level fusion is carried out by concatenating the dominant principal components of faces and palmprints to form a fused feature space. Jing et al., [9] employed Gabor transform for feature extraction and are fused. Xiuqin [10] proposed a multimodal biometric system using face and ear at feature level. Here kernel discriminant analysis is employed as feature extraction method to obtain the features of face and ear independently, which is then concatenated to form the single feature vector. Rattani and Tistarelli [11] proposed a multimodal biometric system of iris and face in which SIFT features of individual modalities are extracted and concatenated to form the fused feature vector. Ross and Govindarajan [14] proposed a multimodal biometric system using face and hand geometry at feature level. Here, face is represented using PCA and LDA while 32 distinct features of hand geometry are extracted and then concatenated to form a fused feature vector. Then sequential feed forward selection is employed to select the useful features from the fused feature vector. Thus from the literature, it is observed that, the feature level fusion is performed by doing feature concatenation.

A match score level fusion consolidates the match scores output by different biometric traits. Apart from the raw data and feature vectors, the match scores contain the richest information about the input pattern. Also it is relatively easy to access and combine the scores generated by different biometric matchers (Ross et al., [16]). Hence, the majority of the works reported on multimodal biometrics are confined to a score level fusion. Score level fusion techniques can be divided into three different categories viz., transformation based methods, classifier based methods, and density based methods. In transformation based methods, scores obtained from different modalities are normalized so that, they lie in the same range. Kittler et al.,[17] proposed a theoreticalframework for transformation based score level fusion approach such as sum rule, median rule, min rule, max rule and product rule. The combination of the scores from three different modalities such as face (frontal and profile) and speech indicates the supreme performance of the sum rule. Snelick et al.,[18] proposed a different normalization schemes for transformed based score level fusion and experimental results indicates that Min-Max normalization scheme is more efficient than all other normalization schemes such as decimal scaling, median, double sigmoid, and tanh normalization scheme. Wang et al., [19] proposed weighted sum rule, where weights are calculated depending on the individual performance of the modalities. In a classifier based methods, a pattern classifier is used to indirectly learn the relationships between the vectors of match scores provided by the different biometric matchers. Hence, the vector of match scores is treated as a feature vector which is then classified into one of the two classes: genuine and imposter. Based on the training set of match scores from genuine and imposter classes, the classifier learns a decision boundary between two classes. Several classifiers have been used to consolidate the match scores of multiple matchers to arrive at a decision. Brunelli and Falavigna [20] uses a hyper BF network to combine matchers based on voice and face features. Chatzis et al., [21] use classical K-means clustering, fuzzy clustering and 
International Journal of Computer Science \& Engineering Survey (IJCSES) Vol.9, No.1/2/3, August 2018

median radial basis function (RBF) for fusion at match score level. Yacoub et al., [22] evaluate a number of classification schemes for fusion of match scores from multiple modalities, including support vector machine (SVM) with polynomial and Gaussian kernels, decision trees, multilayer perceptron, fishers linear discriminant analysis (FLDA) and Bayesian classifier. Wang et al., [23] employ FLDA and a neural network classifier with RBF to classify the scores from face and iris modalities into genuine and imposter classes. Ross and Jain [24] proposed the use of decision trees and FLDA for combining the match scores of face, fingerprint and hand geometry modalities. In density based methods, the densities of genuine and imposter scores are estimated by parametric and non-parametric methods. Snelick et al., [18] use a parametric approach to estimate the conditional densities of the match scores from different modalities. Here score densities are assumed to follow a Gaussian distribution and finally classification is carried out using Bayes rule. Jain et al.,[25] proposed the use of Parzen windowbased non parametric density estimation method to estimate the conditional density of genuine and imposter scores. Prabhakar and Jain [26] proposed to perform score fusion using non parametric approach based on joint multivariate densities. Then, based on the joint densities, the posterior probabilities are computed using Bayes rule. Dass et al., [27] proposed a generalized density estimation scheme which can be used to analyze both continuous and discrete scores. Nandakumar et al.,[28] proposed a density estimation scheme using likelihood ratio (LR), where gaussian mixture model (GMM) is used to accurately estimate the underlying density of genuine and imposter scores and finally NeymanPearson rule is employed to make the final decision. On the other hand, in decision level fusion, the decision outputs by individual modalities are combined. This type of fusion is preferred because many commercial off-the-self (COTS) biometric matchers provide access only to the final recognition decision. In literature, lot of approaches at this level are proposed such as AND and OR rules (Daughman, [29]), majority voting (Lam and Suen, [30], weighted majority rule [31], Bayesian decision (Ross et al., [16]) and Dumpster-Shafer theory of evidence (Ross et al., [16]). As a decision level fusion involves very abstract level of information, it is less preferred in fusion of different modalities in designing multimodal biometric systems.

In [32] Galbally et al, presented a software-based fake detection method that canbe used in multiple biometric systems to detect different types of fraudulent access attempts forenhancing biometric recognition frameworks security through having aliveness assessment in a fast, user friendly and nonintrusive. The proposed method was tested and evaluated using publicly availabledata sets of fingerprint, iris and face [32]. He et al. [33] trained a set of adaptive Gabor filters using particleswarm optimization to achieve the proper Gabor basic functions for each modality. Next, it was projected to a single-scalar score via a trained, supported, vector regression model for a final decision. Facial Recognition Technology database-FAFB, CASIA-V3- Interval and FVC2004-DB2 a datasets were combined to form a large-scale dataset of face, iris and fingerprint biometrics [34]. In [34] Raja et al.,proposed a face, periocular and iris multimodal biometric authentication system, where the proposedsystem was tested on Samsung Galaxy S5 smartphone and Samsung Galaxy Note 10.1 tablet. Menotti etal.,[35] assumed that the sensor had a very limited knowledge about biometric spoofing in order to deriveoutstanding spoofing detection systems for iris, face and fingerprint. Two deep learning approaches wereutilized: The first involves learning suitable convolutional network architectures for each domain, whilethe second concentrates on learning the weights of the network via back-propagation. The researchersconsidered nine biometric spoofing benchmarks and learn deep representations for each benchmark bycombining and contrasting the two learning approaches [10].

\section{Proposed MODEL}

In this section, we propose a multimodal biometric system of Face and fingerprint by integrating the information at feature level and decision level. Initially, SIFT and Gabor features are extracted from both face and fingerprint of a person and are individually studied for their 
International Journal of Computer Science \& Engineering Survey (IJCSES) Vol.9, No.1/2/3, August 2018 identification accuracies. Later, the fusion of the biometric traits is recommended at feature level using the best representation, which is subsequently classified using fusion classifier of KNN, NB, SVM and RBF.

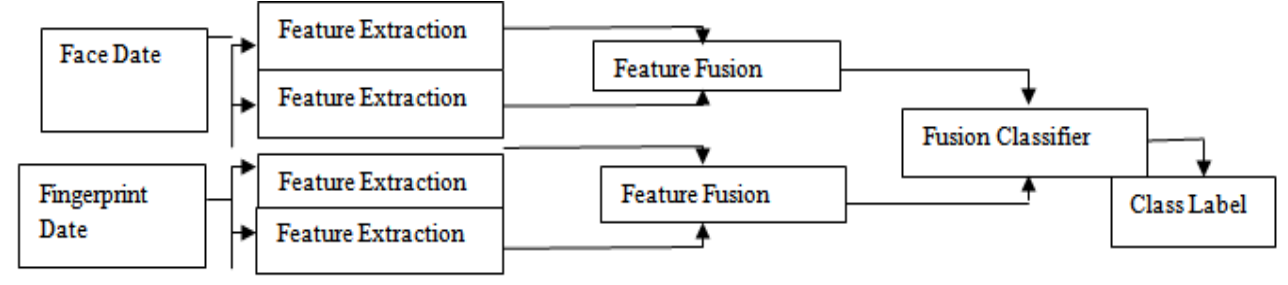

Figure 1: shows the block diagram of proposed system

\section{Feature Level Fusion}

In proposed system, for given face and fingerprint images we extracted Gabor Filter responses and SIFT features.

\subsection{GABOR Filter RESPONSES}

Texture analysis using filters based on Gabor functions falls into the category of frequency-based approaches. These approaches are based on the premise that the texture is an image pattern containing a repetitive structure that can be effectively characterized in a frequency domain, specificallyin Fourier domain. One of the challenges, however, of such an approach is dealing with the trade-off between the joint uncertainty in the space and frequency domains. Meaningful frequency based analysis cannot be localized without bound. An attractive mathematical property of Gabor functions is that they minimize the joint uncertainty in space and frequency. They achieve the optimal trade-off between localizing the analysis in the spatial and frequency domains. Using Gabor filters to analyze texture appeals from a psycho-visual perspective as well. The texture analysis is accomplished by applying a bank of scale and orientation selective Gabor filters to an image (Newsam and Kamath, [36]. These filters are constructed as follows. A twodimensional Gabor function $\mathrm{g}(\mathrm{x} ; \mathrm{y})$ and its Fourier transform $\mathrm{G}(\mathrm{u} ; \mathrm{v})$ can be written as:

$$
g(x, y)=\left(\frac{1}{2 \pi \sigma_{x} \sigma_{y}}\right) \exp \left[-\frac{1}{2}\left(\frac{x^{2}}{\sigma_{x}^{2}}+\frac{y^{2}}{\sigma_{y}^{2}}\right)+2 \pi i W x\right]
$$

and

$$
G(u, v)=\exp \left\{-\frac{1}{2}\left[\frac{(u-W)^{2}}{\sigma_{u}{ }^{2}}+\frac{v^{2}}{\sigma_{v}{ }^{2}}\right]\right\}
$$

where $i=\sqrt{-1}, \sigma_{u}=1 / 2 \pi \sigma_{x}$ and $\sigma_{v}=1 / 2 \pi \sigma_{y}$ control the tradeoff between spatial and frequency resolution, and $\mathrm{W}$ controls the modulation. A class of self-similar functions referred to as Gabor wavelets is now considered. Let $\mathrm{g}(\mathrm{x}, \mathrm{y})$ be the mother wavelet. A filter dictionary can be obtained by appropriate dilations and translations of $\mathrm{g}(\mathrm{x}, \mathrm{y})$ through the generating function.

$$
g_{r s}(x, y)=a^{-s} g\left(x^{\prime}, y^{\prime}\right), a>1, s \in 0, \ldots \ldots . S-1, r \in 1, \ldots . . R
$$

where $x^{\prime}=a^{-s}(x \cos \theta+y \sin \theta) \quad$ and $y^{\prime}=a^{-s}(-x \sin \theta+y \cos \theta)$ with $\theta=(r-1) \pi / R$.

The indices $r$ and $s$ indicate the orientation and scale of the filter respectively. $R$ is the total number of orientations and $\mathrm{S}$ is the total number of scales in the filter bank. While the size of the filter bank is application dependent, it shall be noticed later in experimentation that a bank of 
International Journal of Computer Science \& Engineering Survey (IJCSES) Vol.9, No.1/2/3, August 2018

filters tuned to combinations of $0,2,4,6,8$ and 10 scales, and different orientations, at 22.5 degree intervals are sufficient for analysis.

\subsection{SCALE INVARIANT FEATURE Transform (SIFT)}

The Principal component analysis (PCA) and 2D principalcomponent analysis (2D PCA) are some of the algorithmsused for extracting face and fingerprint information. Thesealgorithms are sensitive to light, expression and pose, etcetera. To overcome these problems, Scale Invariant FeatureTransform (SIFT) was introduced to be used for featureextraction. SIFT method has the advantages of rotation invariance, scale invariance has strong robustness for occlusion problem and noise and affine invariance. [36] SIFTis an algorithm in machine vision used to extract specificfeatures of images for applications such as matchingvarious views of an object and identifying objects. During thefeature extraction the images is pre-processed to obtain betterperformance by reducing the noise. The image is thensubjected to the scale space extrema detection by use ofcascade filtering approach to identify thelocations of thecandidate key points which are invariant to scale changes ofthe image. Key point localization is then performed on theimage to remove all the unrealistic key points that is those with low contrast or is poorly localized along an edge. The image isthen finally assigned a consistent orientation based on localimage properties in order to achieve an image that is invariantto image rotation. SIFT mainly includes four steps which are scale-spaceextrema detection, removal of unreliable key points, orientation assignment and matching. [15] Scale spacedetection of extreme value in space scale: In SIFT, scaletransformation is done by Gaussian convolute on and the descriptor, $\mathrm{L}(\mathrm{x}, \mathrm{y}, \sigma)$ of input image $(\mathrm{I}(\mathrm{x}, \mathrm{y}))$ in the differencescale can be expressed by the equation (3).

$$
L(x, y, \sigma)=(G(x, y, \sigma)) * I(x, y)
$$

Where $\sigma$ is scale factor and Gaussian convolution kernel $(G(x, y, \sigma))$ is given

$$
(G(x, y, \sigma))=\frac{1}{2 \pi \sigma^{2}} e^{-\left(x^{2}+y^{2}\right) / 2 \sigma^{2}}
$$

Where $\sigma$ is scale factor and Gaussian convolution kernelafter the convolution, the calculated image is a Gaussianimage. $\mathrm{L}(\mathrm{x}, \mathrm{y}, \sigma)$. The image $\mathrm{I}(\mathrm{x}, \mathrm{y})$ zoom with $\sigma$, and thesmoothness of the image would change with the change of $\sigma$,and then a series of scale image could beobtained. [15]According to those scale images, the extreme point (keypoints (interest points)) will be detected while the filtering outkey-points: The location of key-point is considered to filter outthe key-points which are sensitive to noise or have no edgeeffect. So for that Taylor quadratic expansion, DoG (x, y, and $\sigma)$ can delete the extreme points which have lower contrast and the value of Hessian vector and the ratio of determinantcan reduce the edge effect.

$$
\begin{aligned}
& D(x, y, \sigma)=(G(x, y, k \sigma)-G(x, y, \sigma)) * I(x, y=L(x, y, k \sigma)- \\
& L(x, y, \sigma)
\end{aligned}
$$

\section{DeCision LeVel Fusion}

The extracted features are fed into fusion classifier of KNN, SVN, Naïve bayes and Radial basis function. Here we combine the decision of all the classifiers.

\subsection{K-NEAREST NeIghbour Algorithm Classifier}

K-nearest neighbour algorithm (k-NN) is a type of instancebased learning algorithm whereby, the function is onlyestimated locally while all computations are done duringclassification. K-NN classifies data entity based on the closesttraining data in the feature space. In thisalgorithm an 
International Journal of Computer Science \& Engineering Survey (IJCSES) Vol.9, No.1/2/3, August 2018

entityclassification is based on majority vote of its neighbours, and the test entity ( $\mathrm{t}$ ) is assigned to the class which is mostcommon among its $\mathrm{k}$ nearest neighbours ( $\mathrm{k}$ refers to a smallpositive integer). [37] Although there is no need for training in this algorithm, the neighbours may be regarded as trainingentities and are chosen from a set of entities for which theirclassification is known. When $\mathrm{k}$ is 1 the test entity is assignedto the class of its nearest neighbour. [38] Feature vectors andclass of the training samples are stored in the training stage ofthe algorithm while in the classification stage, $\mathrm{k}$ is decided bythe user. A test vector is classified by assigning it the labelwhich is most common among the $\mathrm{k}$ training data neighbours.

\subsection{Support Vector MACHINE}

The support vector machine (SVM) is superior to all machine learning algorithms which are based on statistical learning theory. There are a number of publications detailing the mathematical formulation and algorithm development of the SVM [39]. The inductive principle behind SVM is structural risk minimization (SRM), which constructs a hyper-plane between two classes, such that the distance between support vectors to the hyper-plane would be maximum. In order to deal with non-linearly separable classes, the input data are first mapped using a kernel to a higher dimensional space in SVM. The radial basis function (RBF) kernel is popularly used in SVM. Object based SVM is implemented using an open source library called Libsvm (version 2.91). It was found that a parameter U influenced the classification accuracy using the RBF kernel. There are two parameters while using RBF kernels: $\mathrm{C}>0$ is the penalty parameter of the error term. $\hat{\mathrm{U}}=$ kernel parameter. It is not known beforehand which $\mathrm{C}$ and $\hat{\mathrm{U}}$ are the best for one problem; consequently some kind of model selection (parameter search) must be done. The goal is to identify good $(\mathrm{C}, \hat{\mathrm{U}})$ so that the classifier can accurately predict unknown data (i.e., testing data). In our case we found it as $\mathrm{C}=2.0, \hat{\mathrm{U}}=2.0$.

\subsection{RAdial BaSis FunCtion NeURAL NETWORKS}

The construction of a RBF NN, in its most basic form, involves three layers with entirely different roles. The input layer is made up of source nodes (sensory units) that connect the network to its environment. The second layer, the only hidden layer in the network, applies a nonlinear transformation from the input space to the hidden space; where hidden space is in general of high dimensionality. The output layer is linear, supplying the response of the network to the activation pattern applied to the input layer. Following parameters are considered while implementing RBF ANN, $f$ Number of input nodes $=7 f$ Number of output nodes $=9 f$ Number of hidden layers $=1 f$ Number of nodes in each hidden layer $=8 f$ Learning rate $=0.85 f$ Momentum $=0.5 f$ Normalization factor for patterns $=255$.

\subsection{NAIVE BAYES}

Naive Bayes [19] is a successful classifier based upon the principle of Maximum A Posteriori (MAP). Given a problem with $\mathrm{K}$ classes $\{\mathrm{C} 1, \ldots, \mathrm{CK}\}$ with so-called prior probabilities $\mathrm{P}(\mathrm{C} 1)$, . $\ldots, \mathrm{P}(\mathrm{CK})$, we can assign the class label $\mathrm{c}$ to an unknown example with features $\mathrm{x}=(\mathrm{x} 1, \ldots, \mathrm{xN}$ ) such that $\mathrm{c}=\operatorname{argmax}_{\mathrm{c}} \mathrm{P}(\mathrm{C}=\mathrm{cll} \mathrm{x} 1, \ldots, \mathrm{xN})$, that is choose the class with the maximum a posterior probability given the observed data. This aposterior probability can be formulated, using Bayes theorem, as follows: $\mathrm{P}(\mathrm{C}=\mathrm{c} \| \mathrm{x} 1, \ldots, \mathrm{xN})=\mathrm{P}(\mathrm{C}=\mathrm{c}) \mathrm{P}(\mathrm{x} 1, \ldots, \mathrm{xN} \| \mathrm{C}=\mathrm{c}) \backslash \mathrm{P}(\mathrm{x} 1, \ldots, \mathrm{xN}) . \mathrm{As}$ the denominator is the same for all classes, it can be dropped from the comparison. Now, we should compute the so-called class conditional probabilities of the features given the available classes. This can be quite difficult taking into account the dependencies between features. The naive bayes approach is to assume class conditional independence i.e. $\mathrm{x} 1, \ldots, \mathrm{xN}$ are independent given the class. This simplifies the numerator to be $\mathrm{P}(\mathrm{C}=\mathrm{c}) \mathrm{P}(\mathrm{x} 1 \| \mathrm{C}=\mathrm{c}) . . \mathrm{P}(\mathrm{xN} \| \mathrm{C}=\mathrm{c})$, and then choosing the class $\mathrm{c}$ that maximizes this value over all the classes $\mathrm{c}=1, \ldots, \mathrm{K}$. Clearly this 
International Journal of Computer Science \& Engineering Survey (IJCSES) Vol.9, No.1/2/3, August 2018

approach is naturally extensible to the case of having more than two classes, and was shown to perform well inspite of the underlying simplifying assumption of conditional independence.

\section{Results}

In this section, we present the experimental results obtained for the proposed multimodalbiometric indexing system based on feature level fusion of Face and fingerprints. The proposed indexing model is tested on our own database. In this work, 3000 face and fingerprint images from 300 users are considered for experimentation.In order to evaluate the performance of the proposed classification system, Gabor and SIFTfeatures are extracted from both face and fingerprints.

The system was trained using 70\%, 50\%, and 30\% samples per user and was tested with the remaining samples per user respectively. Table 1 shows the summary of the individual and fusion classifier recognition accuracy under varying database size of face using different representatives. Further, the feature vectors of different representations are concatenated serially considering two at a time to obtain new feature vectors are tabulated in Table 1 to Table 3 . Table 2 shows the summary of the individual and fusion classifier recognition accuracy under varying database size of fingerprint using different representatives. Table 3 shows the summary of the individual and fusion classifier recognition accuracy under varying database size of fusion of fingerprint and face using different representatives. From accuracy tables it is observed that the feature level fusion of face and fingerprint and fusion of various classifiers outperforms the face and fingerprints when they are considered individually. Hence this fused feature vector is recommended for the proposed multimodal biometric system

Table 1: Shows the accuracy of Face database

\begin{tabular}{|c|c|c|c|c|c|c|}
\hline Features & $\begin{array}{l}\text { Training } \\
\text { Percentage }\end{array}$ & KNN & $\begin{array}{l}\text { Naïve } \\
\text { Bayes }\end{array}$ & RBN & SVM & FUSION \\
\hline \multirow{3}{*}{ Gabor } & 70 & 85.6 & 83.5 & 84.5 & 85.5 & 90.6 \\
\hline & 50 & 78.5 & 75.6 & 76.5 & 78.62 & 85.5 \\
\hline & 30 & 72.6 & 60.5 & 70.4 & 71.55 & 80.6 \\
\hline \multirow{3}{*}{ SIFT } & 70 & 84.5 & 81.2 & 82.3 & 83.6 & 89.5 \\
\hline & 50 & 76.5 & 74.56 & 74.6 & 76.63 & 83.5 \\
\hline & 30 & 70.5 & 68.6 & 66.6 & 68.9 & 76.5 \\
\hline \multirow{3}{*}{ Gabor+SIFT } & 70 & 87.5 & 84.56 & 85.56 & 86.6 & 91.47 \\
\hline & 50 & 79.5 & 77.96 & 77.34 & 76.63 & 86.77 \\
\hline & 30 & 73.5 & 70.69 & 69.32 & 69.9 & 81.44 \\
\hline
\end{tabular}


International Journal of Computer Science \& Engineering Survey (IJCSES) Vol.9, No.1/2/3, August 2018

Table 2: Shows the accuracy of Fingerprint database

\begin{tabular}{|c|c|c|c|c|c|c|}
\hline & Features & KNN & $\begin{array}{l}\text { Nä̈ve } \\
\text { Bayes }\end{array}$ & RBN & SVM & FUSION \\
\hline \multirow{4}{*}{ Gabor } & $\mathbf{7 0}$ & 84.3 & 81.8 & 83.6 & 85.45 & 91.8 \\
\cline { 2 - 7 } & $\mathbf{5 0}$ & 76.5 & 73.88 & 74.2 & 76.7 & 84.98 \\
\cline { 2 - 7 } & $\mathbf{3 0}$ & 69.8 & 67.3 & 68.4 & 70.6 & 78.45 \\
\hline \multirow{3}{*}{ SIFT } & $\mathbf{7 0}$ & 84.77 & 81.98 & 82.2 & 84.53 & 90.5 \\
\cline { 2 - 8 } & $\mathbf{5 0}$ & 75.5 & 72.5 & 77.4 & 74.6 & 83.97 \\
\cline { 2 - 8 } & $\mathbf{3 0}$ & 68.4 & 62.6 & 71.23 & 68.42 & 75.11 \\
\hline \multirow{3}{*}{ Gabor+SIFT } & $\mathbf{7 0}$ & 86.77 & 83.98 & 84.21 & 86.53 & 92.54 \\
\cline { 2 - 7 } & $\mathbf{5 0}$ & 77.32 & 74.51 & 78.98 & 77.88 & 85.07 \\
\cline { 2 - 7 } & $\mathbf{3 0}$ & 70.4 & 65.3 & 70.44 & 69.67 & 79.31 \\
\hline
\end{tabular}

Table 3: Shows the accuracy of Fusion of Face and Fingerprint database

\begin{tabular}{|c|c|c|c|c|c|c|}
\hline & Features & KNN & $\begin{array}{l}\text { Naïve } \\
\text { Bayes }\end{array}$ & RBN & SVM & FUSION \\
\hline \multirow{3}{*}{ Gabor } & 70 & 90.14 & 87.3 & 87.6 & 89.4 & 95.65 \\
\hline & 50 & 84.52 & 80.97 & 82.9 & 83.2 & 89.23 \\
\hline & 30 & 79.5 & 74.63 & 75.8 & 77.2 & 83.44 \\
\hline \multirow{3}{*}{ SIFT } & 70 & 88.5 & 88.12 & 88.3 & 89.6 & 94.87 \\
\hline & 50 & 83.5 & 82.56 & 84.6 & 83.63 & 88.74 \\
\hline & 30 & 78.5 & 76.99 & 74.6 & 76.63 & 81.22 \\
\hline \multirow{3}{*}{ Gabor+SIFT } & 70 & 91.77 & 89.98 & 89.21 & 90.83 & 96.14 \\
\hline & 50 & 85.32 & 82.97 & 84.98 & 85.78 & 89.87 \\
\hline & 30 & 80.4 & 77.3 & 77.88 & 78.7 & 84.78 \\
\hline
\end{tabular}

\section{Conclusion}

A multimodal biometric system based on the integration of face and a fingerprint trait at feature extraction level and decision level was presented. These two traits are the most widely accepted biometrics in most applications. There are also other advantages in multimodal biometric systems, including the easy of use, robustness to noise, and the availability of lowcost, off-theshelf hardware for data acquisition. From a system point of view, redundancy can always be exploited to improve accuracy and robustness which is achieved in many living systems as well. Human beings, for example, use several perception cues for the recognition of other living creatures. They include visual, acoustic and tactile perception. Starting from these considerations, 
International Journal of Computer Science \& Engineering Survey (IJCSES) Vol.9, No.1/2/3, August 2018 this paper outlined the possibility to augment the verification accuracy by integrating multiple biometric traits. In this paper a novel approach has been presented where both fingerprint and face images are processed with compatible feature extraction algorithms to obtain comparable features from the raw data. The reported experimental results demonstrate remarkable improvement in the accuracies by properly fusing feature sets. This preliminary achievement, does not constitute an end in itself, but rather suggests to attempt a multimodal data fusion as early as possible in the processing pipeline. In fact, the real feasibility of this approach, in a real application scenario, may heavily depend on the physical nature of the acquired signal. The experimental results demonstrate that fusing information from independent/ uncorrelated sources (face and fingerprint) at the feature level fusion increases the performance as compared to score level. As even in the literature, it is claimed that ensemble of classifier operating on uncorrelated features increases the performance in comparison to correlated features. This work does investigation at feature level and decision level and the results are inspiring.

\section{REFERENCES}

[1] Ko T., 2005. Multimodal biometric identification for large user population using fingerprint, face and iris recognition. Proceedings of the thirty fourth Workshop on Applied Imagery and Pattern Recognition, pp. $218-223$.

[2] Ross A. and A. K. Jain, 2003. Information fusion in biometrics. Journal of Pattern Recognition Letters, vol. 24, no. 13, pp. $2115-2125$.

[3] Ross A. A., K. Nandakumar and A. K., Jain, 2006. Handbook of multibiometrics. Springer.

[4] Heo J., S. G. Kong, B. R. Abidi and M. A. Abidi, 2004. Fusion of visual and thermal signatures with eyeglass removal for robust face recognition. Proceedings of IEEE Workshop on Object Tracking and Classification Beyond the Visible Spectrum in conjunction with CVPR, pp. 94 - 99.

[5] Singh R., M. Vatsa, A. Noore, 2008. Integrated multilevel image fusion and match score fusion of visible and infrared face images for robust face recognition. Journal on Pattern Recognition, vol. 41, no. 3 , pp. $880-893$.

[6] S., A. Gyaourova, G. Bebis and I. Pavlidis, 2004. Infrared and visible image fusion for face recognition. Proceedings of the SPIE Defense and security symposium, vol. 5404, pp. 585 - 596.

[7] Kisku D. R., J. K. Singh, M. Tistarelli and P. Gupta, 2009. Multisensor biometric evidence fusion for person authentication using wavelet decomposition and monotonic decreasing graph. Proceedings of the Seventh International Conference on Advances in Pattern Recognition, pp. 205 - 208.

[8] Yao Y., X. Jing and H. Wong, 2007. Face and palmprint feature level fusion for single sample biometric recognition. Journal of Neurocomputing, vol. 70, no. 7 - 9, pp. $1582-1586$.

[9] Jing X. Y., Y. F. Yao, D. Zhang, J. Y. Yang and M. Li, 2007. Face and palmprint pixel level fusion and kernel DCV - RBF classifier for small sample biometric recognition. Journal on Pattern Recognition, vol. 40, no. 11, pp. 3209 - 3224.

[10] Xuiqin P., X. Xiaona, L. Yong, C. Yongcun, 2008. Feature fusion of multimodal recognition based on ear and profile face. Proceedings of the SPIE, vol. 7127. http://dx.doi.org/10.1117/12.806747.

[11] Rattani A. and M. Tistarelli, 2009. Robust multimodal and multiunit feature level fusion of face and iris biometrics. Proceedings of the Third International Conference on Advances in Biometrics, pp. $960-969$. 
International Journal of Computer Science \& Engineering Survey (IJCSES) Vol.9, No.1/2/3, August 2018

[12] Feng G. K. Dong, D. Hu and D. Zhang, 2004. When faces are combined with palmprints: a novel biometric fusion strategy. Proceedings of the International Conference on Biometric Authentication, LNCS 3072, pp. $701-707$.

[13] Zhou X. and B. Bhanu, 2006. Feature fusion of face and gait for human recognition at a distance in video. Proceedings of the Eighteenth International Conference on Pattern Recognition, pp. 529 - 532.

[14] Ross A. and R. Govindarajan, 2004. Feature level fusion using hand and face biometrics. Proceedings of the SPIE conference on Biometric Technology for Human Identification, pp. 196 204.

[15] Feng G. K. Dong, D. Hu and D. Zhang, 2004. When faces are combined with palmprints:a novel biometric fusion strategy. Proceedings of the International Conference on Biometric Authentication, LNCS 3072, pp. $701-707$.

[16] Ross A. A., K. Nandakumar and A. K., Jain, 2006. Handbook of multibiometrics. Springer.

[17] Kittler, J., Hatef, M., Duin, R. P. W., Matas, J. 1998. On combining classifiers. IEEE Transactions on Pattern Analysis and Machine Intelligence, vol. 20, no. 3, pp. 226 - 239.

[18] Snelick R., U. Uludag, A. Mink, M. Indovina and A. K. Jain, 2005. Large scale evaluation of multimodal biometric authentication using state of the art systems. IEEE Transactions on Pattern Analysis and Machine Intelligence, vol. 27, no. 3, pp. 450 - 455.

[19] Wang Y., T. Tan and A. K. Jain, 2003. Combining face and iris biometrics for identity verification. Proceedings of the Fourth International Conference on Audio and Video Based Biometric Person Authentication, pp. $805-813$.

[20] Brunelli R. and D. Falavigna, 1995. Person identification using multiple cues. IEEE Transactions on Pattern Analysis and Machine Intelligence, vol. 17, no. 10, pp. 955 - 966.

[21] Chatzis V., A. G. Bors and I. Pitas, 1999. Multimodal decision level fusion for person authentication. IEEE Transactions on Systems, Man and Cybernetics, Part A: Systems and Humans, vol. 29, no. 6, pp. $674-680$.

[22] Yacoub S. B., Y. Abdeljaoued and E. Mayoraz, 1999. Fusion of face and speech data for person identity verification. IEEE Transactions on Neural Networks, vol. 10, no. 5, pp. 1065 - 1074.

[23] Wang Y., T. Tan and A. K. Jain, 2003. Combining face and iris biometrics for identity verification. Proceedings of the Fourth International Conference on Audio and Video Based Biometric Person Authentication, pp. $805-813$.

[24] Ross A. and A. K. Jain, 2003. Information fusion in biometrics. Journal of Pattern Recognition Letters, vol. 24, no. 13, pp. $2115-2125$.

[25] Jain A. K., L. Hong, Y. Kulkarni, 1999. A multimodal biometric system using fingerprint, face and speech. Proceedings of the International Conference on Audio and Video Based Biometric Person Authentication, pp. $182-187$.

[26] Prabhakar S. and A. K. Jain, 2002. Decision level fusion in fingerprint verification. Journal on Pattern Recognition, vol. 35, no. 4, pp. $861-874$.

[27] Dass S. C., K. Nandakumar and A. K. Jain, 2005. A principled approach to score level fusion in multimodal biometric systems. Proceedings of the International Conference on Audio and Video Based Biometric Person Authentication, pp.1049 - 1055. 
International Journal of Computer Science \& Engineering Survey (IJCSES) Vol.9, No.1/2/3, August 2018

[28] Nandakumar K., Y. Chen, S. C. Dass, A. K. Jain, 2008. Likelihood ratio based biometric score fusion. IEEE Transactions on Pattern Analysis and Machine Intelligence, vol. 30, no. 2, pp. 342 - 347.

[29] Daugman J., 2002. Combining multiple biometric, Available online at www.cl.cam.ac.uk/ jgd1000/combine/combine.html

[30] Lam L. and C. Y. Suen, 1997. Application of majority voting to pattern recognition.IEEE Transactions on Systems, Man, and Cybernetics - Part A: Systems And Humans, vol. 27, no. 5, pp. $553-568$.

[31] Kuncheva L. I. , 2002. Combining pattern classifier methods and algorithm, Wiley Interscience

[32] Galbally, J.; Marcel, S.; Fierrez, J. 2014. Image quality assessment for fake biometric detection: application to iris, fingerprint, and face recognition. IEEE Trans. Image Process. 23(2), 710-724.

[33] He, F.; Liu, Y.; Zhu, X.; Huang, C.; Han, Y.; Chen, Y. (2014) Score level fusion scheme based on adaptive local Gabor features for face-iris-fingerprint multimodal biometric. J. Electron. Imaging 23(3), 033019

[34] Raja, K.B.; Raghavendra, R.; Stokkenes, M.; Busch, C. 2014. Smartphone authentication system using periocular biometrics. In: 2014 International Conference of the Biometrics Special Interest Group (BIOSIG), pp. 1-8. IEEE.

[35] Menotti, D.; Chiachia, G.; Pinto, A.; Robson Schwartz, W.; Pedrini, H.; Xavier Falcao, A.; Rocha, A.: 2015. Deep representations for iris, face, and fingerprint spoofing detection. IEEE Trans. Inf. Forens. Secur. 10(4), 864-879.

[36] Newsam, S. D. and Kamath, C. 2004. Retrieval using texture features in high resolution multi-spectral satellite imagery. In SPIE Conference on Data Mining and Knowledge Discovery.

[37] M.-L. Zhang and Z.-H. Zhou. ML-KNN: A Lazy Learning Approach to Multi-Label Learning. Pattern Recogn., 40(7):2038-2048, 2007.

[38] X. Hao, X. Tao, C. Zhang, and Y. Hu. An Effective Method to Improve kNN Text Classifier. In Eighth ACIS International Conference on Software Engineering, Artificial Intelligence, Networking, and Parallel/Distributed Computing, volume 1, pages 379-384, 2007.

[39] C. J. C. Burges, “A tutorial on support vector machines for pattern recognition”, Data Mining and Knowledge Discovery, 2 (2), pp. 121-167, 1998.

[40] V.N.Vapnik, "The Nature of Statistical Learning Theory”, Springer-Verlag, New York, 1995. 